\title{
The Modular Active Capacitor for High Power Ripple Attenuation
}

\author{
Vinay BHUS, Jyun LIN, and George WEISS
}

\begin{abstract}
In this paper we propose a modular realization of the active capacitor, where each module is composed of a bidirectional converter and a buffering capacitor. This modular capacitor is a two-terminal device that can automatically adapt itself to different $\mathrm{DC}$ bus voltages, and is meant to filter out a wide range of low frequency ripples. Our plug-and-play control approach means that the modular active capacitor can be directly connected to the DC bus without any extra connections, just like a passive capacitor. The proposed modular design improves the overall reliability, providing fault tolerance operation. We investigate the application of our type of active capacitor in DC microgrids, where multiple active capacitors are distributed at different nodes. It is shown that ripple power can be shared in proportion to the storage capacity of the active capacitors without any communication between them. We also offer a mathematical stability analysis of a DC microgrid in which the admittances of all the devices satisfy a certain condition that is less restrictive than being positive-real (and which is satisfied by our proposed design). The modular active capacitor concept has been verified in simulations and experiments.
\end{abstract}

Index Terms-Active capacitor, active power filtering, interleaving modular converter, (twisted) positive-real, ripple attenuation.

\section{INTRODUCTION}

$\mathrm{I}^{2}$ applications ranging from electric vehicle chargers to gridtied inverters, there are several stages of power conversion. At a DC stage, a bank of "DC-Link" capacitors is normally used to filter out low frequency ripple, high frequency switching noise and to provide energy storage. Further examples where filtering capacitors are needed include single phase power factor compensator (PFC) power supplies, photovoltaic systems, adjustable speed drives, uninterruptible power supplies, power supplies for pulsed load [1], [16] and LED lighting systems. The DC-Link capacitors employed in such capacitor banks are usually bulky electrolytic ones that have a limited life span and can break down easily [2], [3]. An alternative are the very reliable film capacitors, but these are bulky and expensive.

Manuscript received October 21, 2020; revised January 26, 2021; accepted March 13, 2021. Date of publication September 30, 2021; date of current version September 8, 2021. This work was supported by a grant from the Momentum Fund managed by Ramot, the technology transfer arm of Tel Aviv University.

All authors are with the School of Electrical Engineering, Tel Aviv University, Ramat Aviv 69978, Israel (e-mail: vinaybhus@mail.tau.ac.il; junlin@post.tau.ac.il; gweiss@tauex.tau.ac.il).

Digital Object Identifier 10.24295/CPSSTPEA.2021.00024

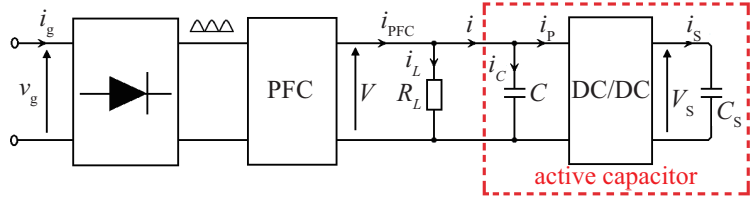

Fig. 1. Typical setup of an AC/DC power supply containing a PFC and a DCLink capacitor $C$. The load $R_{L}$ may represent a second stage $\mathrm{DC} / \mathrm{DC}$ converter leading to the power consumer. Also shown is an active capacitor connected to the DC bus.

Many active power decoupling circuits, also called active capacitors (or ripple eliminators) have been proposed to replace the DC-Link capacitors, see [4], [5], [15]. These circuits are based on the observation that the energy fluctuations in a DC-Link capacitor, caused by the DC bus disturbances, are tiny compared to the actual stored energy in the capacitor [10]. The basic idea is to transfer these "tiny" energy fluctuations to a buffering capacitor $C_{\mathrm{S}}$ via a bidirectional converter, see the reviews [6]-[9]. The whole circuit may have the equivalent effect to that of a capacitor that is much larger than $C_{\mathrm{S}}$. An application example of an active capacitor employed to stabilize the output voltage of a PFC is shown in Fig. 1.

The realizations of active capacitors can be classified into two main categories: The first is that the active capacitor is tightly coupled with the power source in both hardware and software, resulting in various multi-port converter topologies with buffering capabilities, such as in [11]-[13]. These type of realizations have a sophisticated combined controller for the two parts, see [14], [15]. The second category consists of active capacitors built with the plug-and-play $(\mathrm{PnP})$ feature. They can be connected to the DC bus like a passive component, with only two terminals. However, the decentralized controller is very challenging to design, as there is no communication between the active capacitor and the power source nor the loads; the only information available is the local DC bus voltage. If the DC bus disturbance spectrum is known a priori, a resonant controller such as in [2], [18], [19] can be built easily. For wide range frequency ripple attenuation, $\mathrm{PnP}$ control is much more difficult, see [4], [5], [20]-[23].

This paper presents a modular and high power version of our earlier PnP active capacitor from [4]. Our proposed solution is to replace the DC bus capacitor bank with a modular electronic circuit that behaves like a variable-gain active capacitance multiplier, which are termed as the modular active capacitor (MAC). For our MAC prototype we have chosen the control 
algorithm from [4], which is meant to cover a wide range of low ripple frequencies (hundreds of $\mathrm{Hz}$ ) and is very robust under changing operating conditions (such as load changes on the DC bus). In this algorithm, the target is to regulate the energy in the buffering capacitor, which in turn influences the DC bus voltage indirectly. Since most of the ripple energy on the DC bus is directed into the buffering capacitor $C_{\mathrm{S}}$ (see Fig. 2), we get an almost constant DC bus voltage. We refer to Section III for more details on this control algorithm. Our method features a very good transient performance, when there are sudden voltage variations on the DC bus.

The MAC consists of multiple active capacitor modules (up to 8 in our prototype) working in parallel, in an interleaved manner. The modular structure of our circuit allows the user to add or remove modules from the MAC to support a wide range of current ripples/load power. We investigate its possible application in a DC microgrid, where multiple MACs are placed at different nodes and we would like them to share the total ripple power in proportion to their storage capacities.

Interleaving means operating several power sources in parallel at evenly distributed phases to achieve smooth total power. The interleaving technique in power electronics often means an interconnection of several DC-DC converters that work together in a synchronized manner with relative phase shifts at the switching frequency, to achieve lower ripple currents. This technique is generally used in high power applications, where large currents are processed with tight load transients. Perhaps the earliest reference proposing the interleaving technique is [28]. An in-depth mathematical analysis of distributed and interleaved systems in the time and frequency domains can be found in [29]. More recently, interleaving techniques have been used extensively, in multiphase DC-DC converters in systems like electric vehicles, battery energy storage systems etc, see [24]-[27]. A few advantages of interleaving include i) power distribution among multiple modules; ii) reduced current and voltage ripples at the input and output of the converter; ii) reduced stress on the active/passive components; iii) higher efficiency, faster dynamics and support for a wide range of loads. However, one of the main challenges for successful implementation of interleaving is phase management and current balancing. For optimal ripple cancellation and overall stability, the input current must be distributed equally among all the modules. This is also important to avoid thermally stressing any one module and to prevent excessive currents from flowing through the switches. Usually a current control loop with a current sensor on each phase is used to ensure equal distribution of the input current, see for instance [30]. This adds to the cost of the converter and increases the complexity of the control, especially as the number of phases grows. We propose to operate our MAC in discontinuous conduction mode (DCM) to eliminate the individual module current control loops and the associated current sensors.

We devote considerable attention to the stability analysis of a DC microgrid comprising active capacitors of the type discussed in this paper. Most devices in a microgrid have admittances that are positive-real (for instance, passive devices).

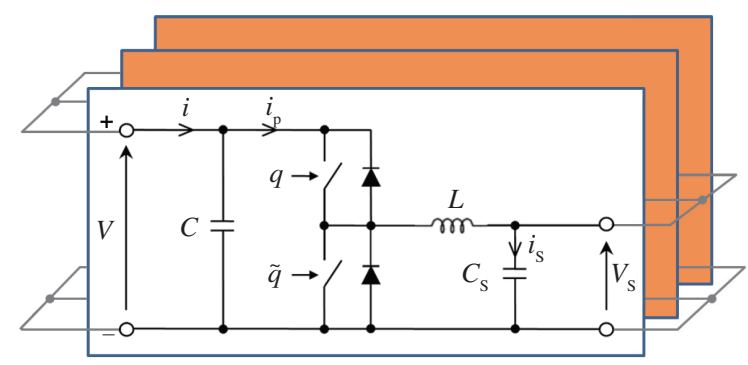

Fig. 2. A MAC circuit with three modules. The $V$ and $V_{\mathrm{S}}$ terminals of the converters are connected with each other, and the modules are controlled by a common micro-controller (not shown).

However, the active capacitors working under the control algorithm from [4] do not have positive-real admittance $Y$. Instead, they satisfy a weaker requirement: $Y(i \omega)$ stays in a rotated right half-plane for all $\omega>0$. Moreover, $Y$ has no unstable poles. We call such transfer functions twisted positive-real. We show that the parallel connection of several devices with twisted positive-real admittance leads to a stable circuit.

The rest of the paper is organized as follows: Section II explains the basic idea behind the proposed MAC. Section III is about the control algorithm. The stability analysis is in Section IV. Section V explains the hardware setup of the MAC and finally Sections VI and VII give the experimental and simulation results.

\section{INTRODUCTION TO THE INTERLEAVED MAC}

The structure of MAC is based on stacking multiple active capacitor modules in a master-slave configuration where one module serves as the master. They are all controlled by a common controller. Each module of the MAC consist of a bidirectional buck converter, with a DC bus filtering capacitor $C$ for high frequency switching noise, and a buffering capacitor $C_{\mathrm{S}}$, as shown in Fig. 2. The input and output terminals of all the converters are connected together, hence the total DC bus and buffering capacitors, $C_{\text {tot }}$ and $C_{\mathrm{S} \text { tot, }}$ are $N$ times $C$ and $C_{\mathrm{S}}$ respectively, where $N$ is the number of modules in the MAC. The voltage sensors for the DC bus voltage $V$, buffering capacitor voltage $V_{\mathrm{S}}$ and the auxiliary power circuitry are only present on the master module. The auxiliary power circuitry is essential for plug-and-play operation of the MAC, as it takes the power from the DC bus and generates different voltage levels that are necessary to power the electronics (drivers, switches, sensors, etc) on the master and slave boards.

The steady state $V-Q$ characteristic curve of the proposed MAC is shown in Fig. 3 where $Q$ denotes the charge accumulated in the MAC (the integral of the current, minus losses). This plot is nonlinear: We have an almost flat region for $Q \in$ $\left[Q_{\text {min }}, Q_{\text {max }}\right]$; whereas for passive capacitors, the dependence of the voltage $V$ on the charge $Q$ is linear. This graph is an approximation of the one corresponding to a virtual infinite capacitor as introduced in [3].

The first region of operation, where $\mathrm{Q} \in\left[0, Q_{\min }\right]$ is called the start-up or the power up region. In this region the converter 


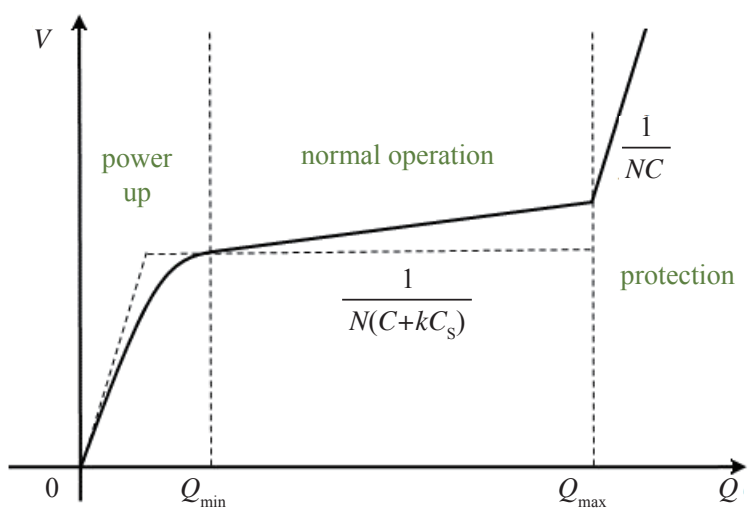

Fig. 3. $V-Q$ characteristic of the MAC at a fixed disturbance frequency.

pre-charges the buffering capacitor $C_{\mathrm{S} \text {, tot, }}$ until its voltage, $V_{\mathrm{S}}$, reaches $V_{\mathrm{S} \text {, min }}$, which is the minimum voltage required for the normal operation of the MAC. The duty cycle increases gradually as $V_{\mathrm{S}}$ rises from 0 to $V_{\mathrm{S} \text {, min }}$. The equivalent capacitance of the MAC in this region starts with $N C$ and increases as the duty cycle increases.

The second region, where $Q \in\left[Q_{\min }, Q_{\max }\right]$ (and hence $V_{\mathrm{S}}$ $\left.\in\left[V_{\mathrm{S}, \min }, V_{\mathrm{S}, \max }\right]\right)$, is the normal operating range of the MAC. In this region, the challenge is to control the MAC in such a way that the charge $Q$ remains in $\left[Q_{\min }, Q_{\max }\right]$ while the voltage $V$ remains almost constant. As a result, the emulated capacitance $C_{\text {eq }}=\mathrm{d} Q / \mathrm{d} V$ is very large and is given by $N\left(C+k C_{\mathrm{S}}\right)$, where $k$ is a variable gain that is frequency dependent; this is explained in Section III.

The third and final region, where $Q>Q_{\max }$, is called the protection region. Here both (upper and lower) switches in all the modules are OFF, hence the equivalent capacitance in this region is $N C$. The value of $V_{\mathrm{S} \text {, max }}$ must be less than $V$ for the MAC to function properly. A state machine that defines the transition of the MAC between these different regions and additional states (not mentioned here) is necessary and must be implemented in the micro-controller [4], [21].

\section{CONTROL OF THE MAC}

\section{A. Overall Control Architecture}

The controller of the MAC is based on [4], where the current-controlled current source type capacitance multiplier shown in Fig. 4(a), has been transformed into a voltage-controlled current source, see Fig. 4(b). Concisely, the idea of the (nonlinear) control in the normal operating range is as follows: We regulate the energy in the buffering capacitor $C_{\mathrm{S}}$, represented by $V_{\mathrm{S}}^{2}$, to follow $V^{2}$ with a gain $k$. For this, the controller adjusts the current $i_{\mathrm{p}}$ (see Figs. 1 and 2) such that, at the ripple frequency, with a good approximation,

$$
V_{\mathrm{s}}^{2}=k V^{2},
$$

where $k$ is a frequency dependent gain. From energy conservation in the converter, it follows that

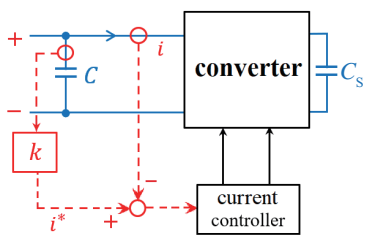

(a)

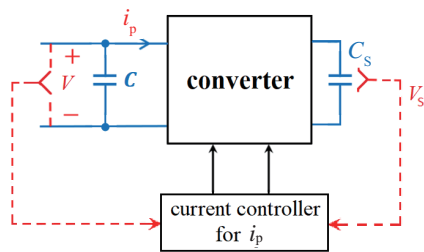

(b)
Fig. 4. (a) A current controlled current source capacitance multiplier with current gain $k>0$ [17]; (b) A buffering capacitor voltage controlled current source active capacitor [4].

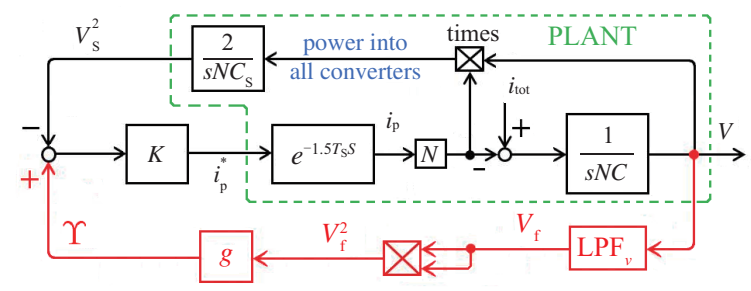

Fig. 5. Overall block diagram of the PnP MAC, adapted from [4]. Here $N$ is the number of modules and $i_{\text {tot }}=N i$ is the total input current.

$$
i_{\mathrm{p}}=\sqrt{k} i_{\mathrm{S}}=\sqrt{k} C_{\mathrm{S}} \dot{V}_{\mathrm{S}}=k C_{\mathrm{S}} \dot{V},
$$

so that $C_{\mathrm{S}}$ appears at the terminals of the active capacitor magnified by $k$. For frequencies well below the ripple frequency, we want $k$ to be much smaller, since a small change in the DC component of $V$ would cause a large change in $V_{\mathrm{S}}$, which we want to avoid. It follows that the equivalent capacitance of the whole MAC is

$$
C_{\mathrm{eq}}=N\left(C+k C_{\mathrm{S}}\right) .
$$

A more detailed representation of the MAC (viewed as a control system) is shown in Fig. 5, following [4]. The low-pass filter $\mathrm{LPF}_{v}$ is only meant to eliminate high frequency noise. The computations and the PWM block are represented by the delay block, where $T_{\mathrm{S}}$ is the sampling and PWM period. For more detailed explanations we refer to [4], but based on what we said here, we can immediately recognize from the diagram that the block $g$ (which is actually a variable structure filter) plays the role of the variable gain $k$ from (1).

The reference for $V_{\mathrm{S}}^{2}$, denoted by $\Upsilon$, is generated based on the fact that the ripple in $V_{\mathrm{S}}^{2}$ should be in phase with $V^{2}$ (see (1) or equation (4) in [4]). In the normal operating range, a second order lead-lag transfer function $g$ is applied to the filtered $V^{2}$ to obtain the reference $\Upsilon$ :

$$
g(s)=a \frac{c^{3} \tau s+1}{c^{2} \tau s+1} \cdot \frac{c \tau s+1}{\tau s+1}
$$

The choice of the parameters for $g$ is delicate as we want to transfer the ripples in the frequency range of interest while keeping the phase of the input impedance close to $-90^{\circ}$, in order to emulate the behaviour of a capacitor. At very low 
frequencies we want the gain of $g$ to be small in order to keep $V_{\mathrm{S}}$ within the normal operating range. At $\mathrm{DC}$ we want the gain to be $g(0)=a=0.5$, so that on average of $V_{\mathrm{S}}^{2}$ remains at the midpoint of $V^{2}$. In the frequency range where we want to suppress the ripple currents the gain of $g$ should be much higher, about 30-35 times compared to that of at low frequencies. In this frequency range, $g(i \omega) \approx a c^{2}$.

To improve the performance of the MAC under sudden load variations, the block $g$ contains also an adaptive gain, as proposed in [4, Section II]: when a sudden change in the DC bus voltage is detected, the gain of $g$ is reduced in high frequencies. This is to avoid large transients that may cause the voltage $V_{\mathrm{S}}$ to get out of the normal operating range.

To generate the current reference $i_{\mathrm{p}}^{*}$, an energy controller $K$ has been designed by using the linearized transfer function from $i_{\mathrm{p}}$ to $V_{\mathrm{S}}^{2}$ around an equilibrium DC bus voltage $V_{0}$ :

$$
\frac{\widehat{V_{\mathrm{S}}^{2}}(s)}{\widehat{i_{\mathrm{p}}}(s)}=\frac{2}{s C_{\mathrm{S}}} V_{0} .
$$

A lag-lead type transfer function $K$ was used as the energy controller in [4]:

$$
K(s)=K_{0} \frac{1+\varepsilon \theta_{\mathrm{s}}}{1+\theta_{\mathrm{s}}}
$$

The gain of the energy controller $K_{0}$ is independent of the number of interleaved modules, as its output $i_{\mathrm{p}}^{*}$ is the reference for $i_{\mathrm{p}}$ for one module.

Depending on the sign of the current reference $i_{\mathrm{p}}^{*}$, all the modules in the MAC are operated either in buck mode or in boost mode in discontinuous conduction mode (DCM). The duty cycles are calculated by equating $i_{\mathrm{p}}^{*} T_{\mathrm{S}}$ to the area of the triangle under the inductor current waveform when the switch is ON. In buck mode $\left(i_{\mathrm{p}}^{*}>0\right)$, the duty cycle for the upper switch (in all the modules) is set to be (see [4])

$$
D=\sqrt{\frac{2 L i_{\mathrm{p}}^{*}}{\left(V-V_{\mathrm{s}}\right) T_{\mathrm{s}}}},
$$

and the lower switch is OFF while allowing current through its freewheeling diode. For the boost mode $\left(i_{\mathrm{p}}^{*}<0\right)$, the duty cycle for the lower switch is set to be

$$
\widetilde{D}=\sqrt{-\frac{2 L\left(V-V_{\mathrm{S}}\right) i_{\mathrm{p}}^{*}}{V_{\mathrm{s}}^{2} T_{\mathrm{S}}}},
$$

and the upper switch is OFF, allowing the current through its freewheeling diode. Note the convenient fact that the control algorithm does not need adjusting when we change the number of modules in the MAC.

\section{B. Controller Tuning}

The ripple currents on the DC bus depend on the sources feeding the bus and the loads connected to the bus. There-

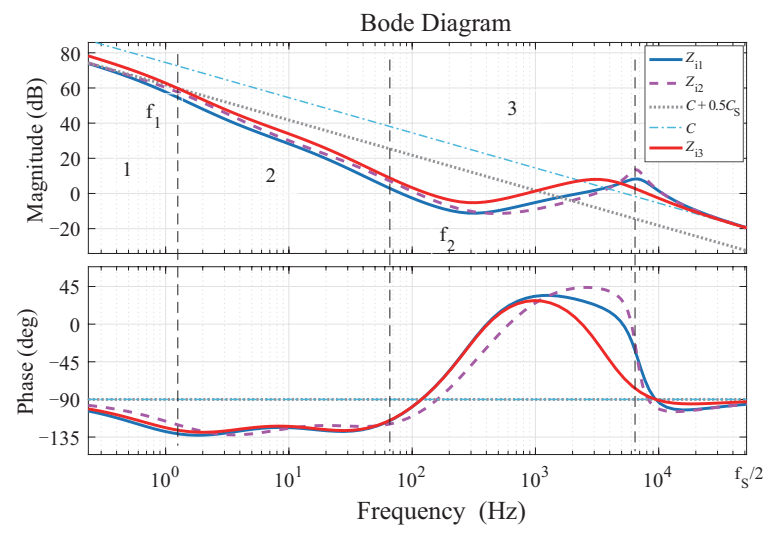

Fig. 6. Bode plots of the input impedances $Z_{1}, Z_{2}$ and $Z_{3}$ for the MAC with three choices of controller parameters, see Subsections III. B and C. The time delay has been approximated by a first order Padé approximation.

fore the frequency range of interest (the working range of the MAC) where we expect the ripple currents to be is application specific. In [4] the controller was tuned to suppress the ripples in the frequency range of 50-700 Hz. In order to suppress the ripples in a higher frequency range, the parameters of $K$ and $g_{2}$ need to be changed. From [4], we have the following input impedance $Z$ of one module of the MAC:

$$
Z(s)=\frac{\frac{1}{s C}}{1+2 g(s) G_{I}(s) V_{0} / s C},
$$

where

$$
G_{I}(s)=\frac{K(s) e^{-1.5 T_{\mathrm{S}} s}}{1+K(s) e^{-1.5 T_{\mathrm{S}} s} 2 V_{0} / s C_{\mathrm{S}}}
$$

is the transfer function of the smaller loop from $\Upsilon$ to $i_{\mathrm{p}}$, see Fig. 5 . In the MAC, the total impedance will be $Z_{\mathrm{i}}(s) / N$, where $N$ is the number of modules. In region 1 at very low frequencies, the impedance of the MAC is mostly capacitive, corresponding to a capacitor with capacitance $C+a C_{\mathrm{S}}$. Region 2 is the transition region, where $f_{1}$ is the breakout frequency, at which the absolute value of the impedance of MAC starts to become smaller than that of the capacitor $C+a C_{\mathrm{S}}$. Region 3 is where we expect the ripples to be. In this region the absolute value of the impedance of the MAC has a local minimum at the frequency $f_{2}$, roughly the bandwidth of the energy controller $K$. Hence, to suppress the ripples in a higher frequency range, we can i) shift the Bode plots of $g$ to the right by decreasing $\tau$; ii) increase the bandwidth of the energy controller by decreasing $\theta$.

Fig. 6 shows three versions of the input impedance $Z$ of the MAC (one module), with $Z_{1}$ calculated using the parameters in Table II of Section V. When both $\tau$ and $\theta$ are decreased by a factor of two, we obtain $Z_{2}$, whose Bode plots are shifted to the right in comparison to $Z_{1}$.

To suppress higher frequencies, the sampling frequency of the controller and the switching frequency of the PWM signals for the switches must be increased. This in turn requires a stronger microprocessor to run the controller, so that the duty cycle control algorithm can be finished within each sampling 
period. Another solution is to partially shift the computations to analog, for example, the current loop can be implemented in critical conduction mode by a flip-flop, or hysteresis control in continuous conduction mode.

\section{Power Sharing}

In the frequency range of interest, i.e., region 3 of the Bode plots in Fig. 6, the input impedance (5) can be simplified:

$$
Z(s) \approx \frac{1}{2 g(s) G_{I}(s) V_{0}}=\frac{1+K(s) 2 V_{0} / s C_{\mathrm{S}}}{2 g(s) K(s) V_{0}} .
$$

If $K(s)$ in (4) is chosen such that $K_{0}$ is proportional to $C_{\mathrm{S}}$, the impedance ratio bewteen MAC $i$ and $j$ can be derived as:

$$
Z_{i}(s): Z_{j}(s)=K_{0, j}: K_{0, i}=1 / C_{\mathrm{S}, i}: 1 / C_{\mathrm{S}, j},
$$

which indicates that the impedance ratio between two active capacitors is similar to that of passive ones. See also the Bode plots of $Z_{1}$ and $Z_{3}$ in Fig. 6 (the red and blue curves), where $C_{\mathrm{S}, 3}$ $=0.5 C_{\mathrm{S}, 1}$ and $K_{0,3}=0.5 K_{0,1}$. Thus, power sharing according to capacity in a wide range of frequencies among multiple MACs can be guaranteed.

\section{DC Microgrid Stability AnAlysis}

Consider a DC microgrid containing several devices, among them, one or more modules of MACs as proposed in this paper. We call this microgrid stable if its impedance $Z$ is stable, i.e., it is bounded and analytic on the open right half-plane $\mathbb{C}_{+}$. If $Z$ is rational, then it is stable if it is proper and all its poles are in the open left half-plane $\mathbb{C}$. . (If a device is nonlinear, then we mean the impedance of its linearization assuming the nominal voltage of the microgrid.)

We have to digress into complex analysis. Recall that a rational transfer function $G$ is called positive-real if $\operatorname{Re} G(s) \geqslant 0$ for all $s \in \mathbb{C}_{+}$. It is well-known that positive-realness in combination with other assumptions implies stability, see for instance [31] for detailed discussion of this and [21, Section IV] for an application to a microgrid containing a $\mathrm{PnP}$ active capacitor of a certain type.

Unfortunately, the impedance of our proposed MAC is not positive-real: we see in Fig. 6 that the phase of $Z(i \omega)$ goes below $-\pi / 2$. However, the impedance $Z$ has another related property that helps us in the stability analysis. Define

$$
\mathscr{W}_{\varphi}=e^{i \varphi} \mathbb{C}_{+},
$$

that is, $\mathscr{W}_{\varphi}$ is obtained by rotating the half-plane $\mathbb{C}_{+}$by the angle $\varphi \in(-\pi / 2, \pi / 2)$. In particular, $\mathscr{W}_{0}=\mathbb{C}_{+}$.

We see from Fig. 6 that the phase of $Z(i \omega)$ for $\omega>0$ is within $\left(-135^{\circ}, 45^{\circ}\right)$, i.e. $Z(i \omega) \in \mathscr{W}_{-\pi / 4}$. Accordingly, the admittance $Y=1 / Z$ satisfies $Y(i \omega) \in \mathscr{W}_{\pi / 4}$ for all $\omega>0$. This can be seen also in Fig. 7 that shows the Nyquist plots of $Y$ for two realizations of the MAC introduced in Subsection III. C. Due to the symmetry of the Nyquist plots, we have that $Y(i \omega) \in \mathscr{W}_{-\pi / 4}$ for all $\omega<0$.

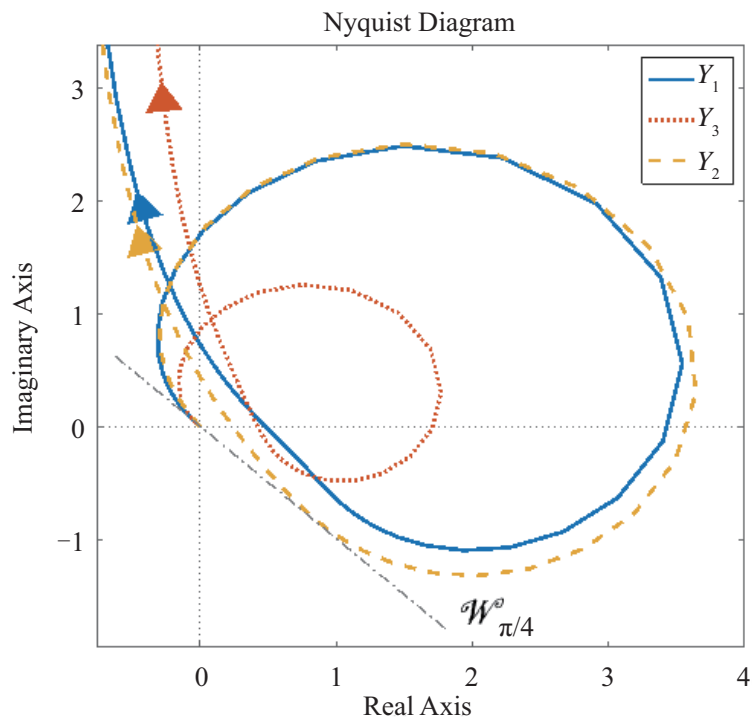

Fig. 7. Nyquist plots of the admittances $Y_{1}, Y_{2}$ and $Y_{3}$ for the MAC. Only the curves corresponding to $\omega>0$ are shown.

Motivated by the above observation, we introduce the following concept: A rational transfer function $G$ is called twisted positive-real with twisting angle $\varphi \in(-\pi / 2, \pi / 2)$ if all its poles are stable (i.e. they are in $\mathbb{C}_{-}$) and

$$
\left\{\begin{array}{l}
G(i \omega) \in \mathscr{W}_{\varphi} \text { for } \omega>0, \\
G(i \omega) \in \mathscr{W}_{-\varphi} \text { for } \omega<0 .
\end{array}\right.
$$

If $G$ is real-rational, meaning that the coefficients in its top and bottom polynomial are real, then the two lines in (6) are equivalent, so that it is enough to assume one of them.

Lemma 4.1: Let $G$ be a real-rational function that is twisted positive-real with twisting angle $\varphi \in(0, \pi / 2)$, and assume that $d=\lim _{\mathrm{s} \rightarrow \infty} G(s) / s$ is finite. Then

$$
G(s) \in \mathscr{W}_{\varphi} \cup \mathscr{W}_{-\varphi} \quad \forall s \in \mathbb{C}_{+} .
$$

Proof: We denote by $G\left(\mathbb{C}_{+}\right)$the image of $\mathbb{C}_{+}$through the function $G$, and by $\partial G\left(\mathbb{C}_{+}\right)$its boundary. We claim that $\partial G\left(\mathbb{C}_{+}\right)$ is contained in the image of $i \mathbb{R}$ (the imaginary axis), together with the image of $\infty$, that is,

$$
\partial G\left(\mathbb{C}_{+}\right) \subset G(i \mathbb{R}) \cup\{G(\infty)\}
$$

This is a consequence of the open map theorem in complex analysis, see for instance [32, Section V], which says that the image of an open set through an analytic function is again open. Hence, if $z \in \partial G\left(\mathbb{C}_{+}\right)$, then $z$ cannot be in the open set $G\left(\mathbb{C}_{+}\right)$, but it can be written as $\lim G\left(s_{n}\right)$ where the sequence $\left(s_{n}\right)$ is in $\mathbb{C}_{+}$. The sequence $\left(s_{n}\right)$ must have s subsequence that converges either to a point $i \omega$ on the boundary of $\mathbb{C}_{+}$(the imaginary axis), or to $\infty$, and from here (7) follows.

It follows from (6) (using that $G(0)=\lim _{\omega \rightarrow 0} G(i \omega)$ must be real) that

$$
G(i \mathbb{R}) \subset \mathscr{W}_{\varphi} \cup \mathscr{W}_{-\varphi} \cup\{0\}
$$




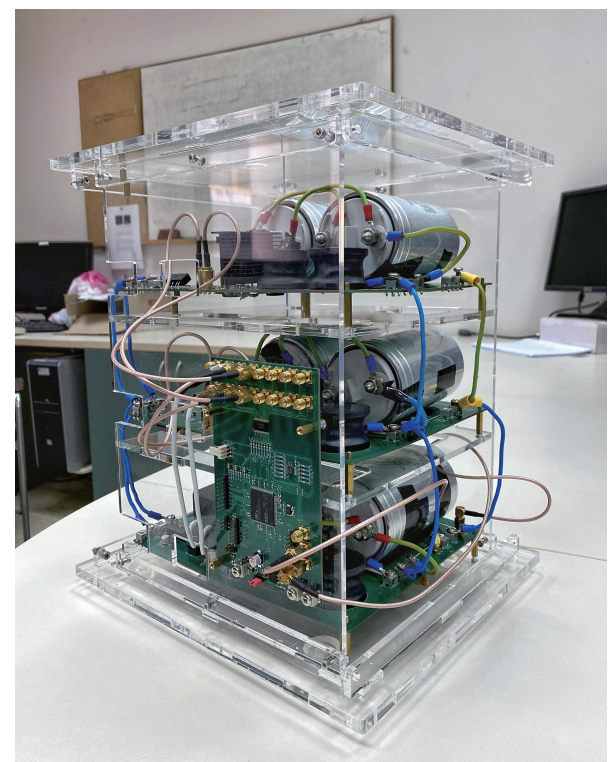

Fig. 8. Our prototype MAC with three modules. The controller is on the vertically standing PCB visible in the front.

Since $G\left(\mathbb{C}_{+}\right)$is a connected set, it follows from (7) and (8) that the domain $\mathscr{D}=\operatorname{int}\left(\mathbb{C} \backslash\left(\mathscr{W}_{\varphi} \cup \mathscr{W}_{-\varphi}\right)\right)$ is either contained in $G\left(\mathbb{C}_{+}\right)$or $\mathscr{D} \cap G\left(\mathbb{C}_{+}\right)=\Phi$. We claim that the first option is not possible. Indeed, it follows from the assumptions on $G$, using partial fractions expansion, that

$$
G(s)=d s+G_{0}(s), \quad d \geqslant 0,
$$

where $G_{0}$ is a stable transfer function. (The fact that $d \geqslant 0$ follows from the fact that if $d \neq 0$ and $\omega>0$ is large, then $G(i \omega)$ $\approx i d \omega$, and this must be in $\mathscr{W}_{\varphi}$.) Hence we conclude that the second option holds, $\mathscr{D} \cap G\left(\mathbb{C}_{+}\right)=\Phi$. Hence the open set $G\left(\mathbb{C}_{+}\right)$ is contained in the interior of the complement of $\mathscr{D}$, and this is precisely what we had to prove.

Consider the parallel connection of $n$ devices with admittances $Y_{1}, Y_{2}, \ldots Y_{n}$. The total impedance of the circuit is

$$
Z_{\mathrm{T}}=\frac{1}{Y_{\mathrm{T}}}, \quad Y_{\mathrm{T}}=Y_{1}+Y_{2}+\ldots+Y_{n} .
$$

We prove a sufficient condition for the stability of $Z_{\mathrm{T}}$.

Proposition 4.2: Let $Y_{1}, Y_{2}, \ldots Y_{n}$ be real-rational functions that are twisted positive-real with twisting angle $\varphi \in(0, \pi / 2)$, and having the property that $\lim _{s \rightarrow \infty} Y_{j}(s) / s$ is finite. Assume that there exists an index $j$ such that $Y_{j}(0)>0$. Then the function $Z_{\mathrm{T}}$ defined by ( 9 ) has no unstable poles.

The assumption $Y_{j}(0)>0$ is natural: it means that there exists at least one DC load in the circuit.

Proof: It is easy to see that the function $Y_{\mathrm{T}}$ from (9) has the same properties that have been assumed for $Y_{j}(1 \leqslant j \leqslant n)$. Hence, according to Lemma 4.1, we have $Y_{\mathrm{T}}(s) \in \mathscr{W}_{\varphi} \cup \mathscr{W}_{-\varphi}$, for all $s \in \mathbb{C}_{+}$. We claim that $Y_{\mathrm{T}}$ has no zeros in the closed right half-plane $\mathbb{C}_{+} \cup i \mathbb{R}$. If $Y_{\mathrm{T}}$ would have a zero in $\mathbb{C}_{+}$, then invoking again the open map theorem, $Y_{\mathrm{T}}\left(\mathbb{C}_{+}\right)$would contain a neighbor-

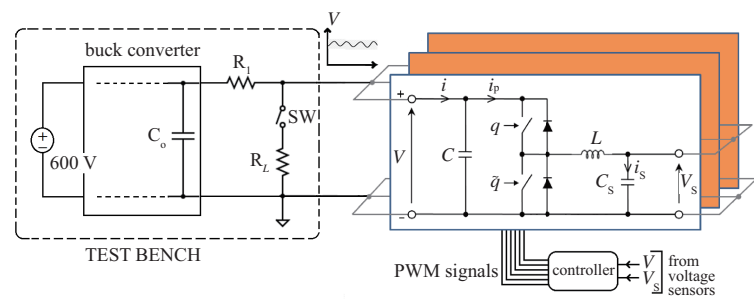

Fig. 9. The experimental setup with the test bench and three interleaved MAC modules.

hood of 0 , in contradiction with Lemma 4.1. Thus, $Y_{\mathrm{T}}$ has no zeros in $\mathbb{C}_{0}$.

For $\omega \in \mathbb{R}$ and $\omega \neq 0$, we cannot have $Y_{\mathrm{T}}(i \omega)=0$ (because $\left.Y_{\mathrm{T}}(i \omega) \in \mathscr{W}_{\varphi}\right)$. Finally, the existence of an index $j$ such that $Y_{j}(0)>0$ implies that $Y_{\mathrm{T}}(0)>0$, finishing the proof of our claim. From this claim, it follows that $Z_{\mathrm{T}}$ does not have any poles in $\mathbb{C}_{+} \cup i \mathbb{R}$.

Note that if the circuit contains at least one capacitor, $Y_{j}(s)=$ $C_{\mathrm{S}}$ (with $C>0$, this satisfies the assumptions in the proposition), then $Y_{\mathrm{T}}(\infty)=\infty$, so that $Z_{\mathrm{T}}$ is proper and hence stable. A similar conclusion holds if the circuit contains at least one resistor, because then $Y_{\mathrm{T}}(\infty)>0$.

\section{Hardware Setup}

The experimental and simulation setup consists of two parts, the test bench and the MAC, see Fig. 9.

The test bench is a model of the DC bus system where random variations of the voltage may occur, due to ripple and also due to the sources and the loads being connected/disconnected. For the purposes of analysis, it is more convenient to examine the effect of sinusoidal voltage variations on the DC bus. We have built a powerful signal generator, that consists of a half bridge buck converter and its control box, fed by a $600 \mathrm{~V} \mathrm{DC}$ power supply. Its duty cycle is set to be:

$$
D=D_{0}+\sum_{k=1}^{3} D_{k} \sin \left(2 \pi f_{k} t\right)
$$

Hence, at the output of the buck converter we have a high voltage DC signal (up to $500 \mathrm{~V}$ ) with a super-position of up to 3 sine waves at different frequencies. The output of the buck converter is connected to the MAC through a small resistor $R_{1}$ and a load resistor $R_{L}$. The resistor $R_{1}$ is necessary in order to prevent the controller of the MAC and the buck converter from competing against each other. Connecting disconnecting $R_{L}$ is used to test the performance of the MAC under sudden load variations.

As a proof of concept, a $400 \mathrm{~V}$ rated modular active capacitor with three active modules working in parallel to handle a high pulsating power of up to $7.5 \mathrm{KW}$ was built, as shown in Fig. 8 . Each module on the MAC prototype can absorb current ripples that correspond to a load power of $2.5 \mathrm{KW}$ fed from a variable DC current source that swings from zero to double the load power (such as a PFC) at different frequencies. The MAC suppresses the ripple and maintains an almost constant voltage with 
TABLE I

MAIN COMPONENT LIST

\begin{tabular}{ll}
\hline \hline Microcontroller & STM32 H743ZI \\
Switches & Transphorm GaN FET TPH3212PS \\
Driver & Silicon Labs SI8230 \\
$C$ & KEMET C4AQIBW5300A3LJ 30 $\mu \mathrm{F}$ \\
$C_{\mathrm{S}}$ & EPOCS B32361A2107 J050 2x100 $\mu \mathrm{F}$ \\
$L$ & Bourns 1140-560K-RC 2x56 $\mu \mathrm{H}$ \\
\hline \hline
\end{tabular}

TABLE II

System Parameters

\begin{tabular}{cc||cc}
\hline \hline$R_{L}$ & $440 \Omega$ & $R_{1}$ & $10 \Omega$ \\
$f_{\mathrm{S}}$ & $100 \mathrm{KHz}$ & $C_{\mathrm{o}}$ & $30 \mu \mathrm{F}$ \\
$K_{0}$ & 0.00035 & $\theta$ & $1 / 2 \pi 400$ \\
$\varepsilon$ & 0.25 & $\tau$ & $1 / 2 \pi 100$ \\
$a$ & 0.5 & $c$ & 5.5 \\
\hline \hline
\end{tabular}

less than $5 \mathrm{~V}$ peak-to-peak ripple on the DC bus, when the ripple frequency ranges from 70 to $300 \mathrm{~Hz}$. The MAC modules are operated at the switching frequency of $100 \mathrm{KHz}$. A list of the main components used in the hardware implementation of the MAC and the control parameters can be found in the Tables I and II.

The major passive power processing components in the MAC are the inductor $L$ and the buffering capacitor $C_{\mathrm{S}}$. The choice of $L$ has to guarantee the DCM operation of the MAC modules. By analyzing the inductor current waveforms in the buck and boost mode, we can write the following condition for the DCM operation:

$$
T_{\mathrm{DCM}}<T_{\mathrm{S}}
$$

where $T_{\mathrm{DCM}}=V / V_{\mathrm{S}} D T_{\mathrm{S}}$ is the time when the inductor discharges and its current drops to 0 . Neglecting the power losses in the MAC modules, $V_{\mathrm{S}} i_{\mathrm{S}}=V i_{\mathrm{p}}$, so that condition (11) can be rewritten as (see also [14]):

$$
L<\frac{V_{\mathrm{S}}^{2}}{V^{2}} \frac{\left(V-V_{\mathrm{S}}\right) T_{\mathrm{S}}}{2 i_{\mathrm{p}}}=\frac{V_{\mathrm{S}}}{V} \frac{\left(V-V_{\mathrm{S}}\right) T_{\mathrm{S}}}{2 i_{\mathrm{S}}} .
$$

This gives us an upper limit for selecting the inductor $L$.

As for $C_{\mathrm{S}}$, recall equation (2) from [4]:

$$
\frac{\mathrm{d}}{\mathrm{d} t} V_{\mathrm{S}}^{2}=\frac{2}{C_{\mathrm{S}}} V i_{\mathrm{p}}
$$

Using the above equation, ignoring the power losses and following a simple computation we can write

$$
C_{\mathrm{S}}=\frac{2 V_{0} \Delta i}{\omega \Delta V_{\mathrm{S}}^{2}},
$$

where $V_{0}$ is the equilibrium DC bus voltage at steady state, $\Delta i=2 i_{\text {amp }}$ is the peak-to-peak value of the ripple current entering the MAC, $\omega$ is the ripple frequency and $\Delta V_{\mathrm{S}}^{2}$ is the maximal allowed excursion of $V_{\mathrm{S}}, \Delta V_{\mathrm{S}}^{2}=V_{\mathrm{S} \text {, } \max }^{2}-V_{\mathrm{S} \text {, min. }}^{2}$. For the proper functioning of the buck converter, we have to take safety margins expressed by numbers $\alpha, \beta \in(0,1)$ such that $V_{\mathrm{S}, \max }=\alpha V_{0}$ and $V_{\mathrm{S}, \text { min }}=\beta V_{0}$. For instance, we may set $\alpha=$ 0.92 and $\beta=0.2$. The previous formula can be rewritten as:

$$
C_{\mathrm{S}}=\frac{2 V_{0} \Delta i}{\omega\left(\alpha^{2}-\beta^{2}\right) V_{0}^{2}}=\frac{2 \Delta i}{\omega\left(\alpha^{2}-\beta^{2}\right) V_{0}} .
$$

This means that the choice of the capacitor $C_{\mathrm{S}}$ depends on the size of the expected current ripple on the DC bus $\Delta i$, the ripple frequency $\omega$ and the equilibrium DC bus voltage $V_{0}$. These parameters are application specific.

For example, let us assume that we use the MAC to stabilize the DC bus after a PFC, as in Fig. 1. The grid voltage and currents are in phase and given by:

$$
v_{\mathrm{g}}=v_{0} \cos \left(\omega_{\mathrm{g}} t\right) \text { and } i_{\mathrm{g}}=i_{0} \cos \left(\omega_{\mathrm{g}} t\right)
$$

which implies via energy conservation that at the output of the PFC we have the current

$$
i_{\mathrm{PFC}}=\frac{v_{0} i_{0}}{2 V}\left(1+\cos \left(2 \omega_{\mathrm{g}} t\right)\right)
$$

Assuming an ideal active capacitor (i.e. $\left|Z_{\mathrm{i}}\left(i 2 \omega_{\mathrm{g}}\right)\right|<<R_{L}$ and $\left.\left|Z_{\mathrm{i}}\left(i 2 \omega_{\mathrm{g}}\right)\right|<<1 / 2 \omega_{\mathrm{g}} C\right)$, we can write:

$$
\left|i_{\mathrm{p}}\right| \approx|i|=\frac{v_{0} i_{0}}{2 V} \frac{R_{L}}{\left|R_{L}+Z_{\mathrm{i}}\left(i 2 \omega_{\mathrm{g}}\right)\right|} \approx \frac{v_{0} i_{0}}{2 V},
$$

where $\left|i_{\mathrm{p}}\right|$ denotes the amplitude of the signal $i_{\mathrm{p}}$, and similarly for $|i|$. Denoting by $P_{\text {grid }}$ the power taken from the grid and using equations (12), (13) and $v_{0} i_{0}=2 P_{\text {grid }}$, we conclude that in this case $C_{\mathrm{S}}$ should be chosen according to

$$
C_{\mathrm{S}}=\frac{2 P_{\text {grid }}}{\omega_{\mathrm{g}}\left(\alpha^{2}-\beta^{2}\right) V_{0}^{2}}
$$

\section{SimUlation VerificATION}

\section{A. Single MAC With Three Modules Connected to the DC Bus}

The following simulation results correspond to the setup shown in Fig. 9. Here the test bench was configured to output a $390 \mathrm{~V}$ DC signal with a $300 \mathrm{~V}$ peak-to-peak disturbance ripple at $100 \mathrm{~Hz}$ in the absence of any DC-Link capacitor. As shown in Fig. 10, when the MAC is connected and reaches the normal operating range, the peak-to-peak ripple on the DC bus is reduced to less than $10 \mathrm{~V}$.

The top subfigure of Fig. 11 shows the interleaved inductor currents of the three individual modules. Comparing the total current (at the bottom of Fig. 11) to the individual phase currents, is it clear that the individual phase currents add up to reduce the ripple in the total converter current of the MAC (as compared to a single module taking the same average current). Each phase in the MAC is working in DCM where the current 

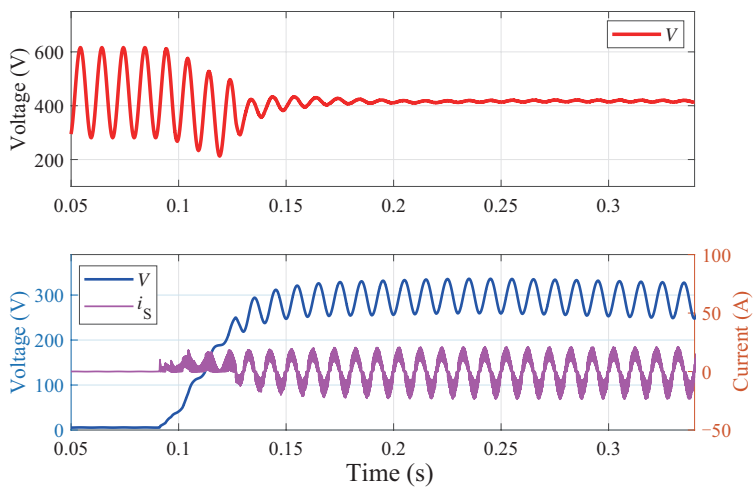

Fig. 10. Simulation results for the MAC starting to operate at $t=0.09 \mathrm{~s}$. The plots of $V, V_{\mathrm{S}}$ and $i_{\mathrm{S}, \text { tot }}$ are arranged from top to bottom. We see that the MAC reaches fast the normal operating range, suppressing the ripple on $V$.
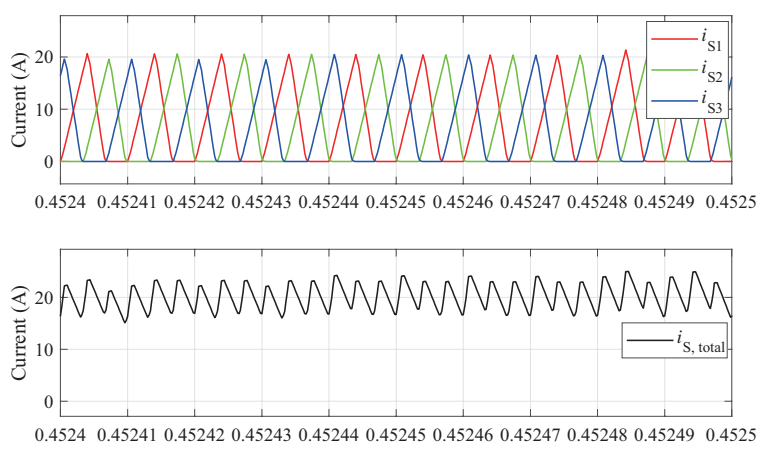

Fig. 11. Simulation results for interleaving, top: zoom in of the three phase individual inductor currents $i_{\mathrm{S} 1}, i_{\mathrm{S} 2}$ and $i_{\mathrm{S} 3}$, bottom (in black): the total current $i_{\mathrm{S} \text {, tot }}$ that is the sum of the individual inductor currents.

pulses are higher compared to the total current that appears to be in continuous conduction mode. The interleaving technique has improved the quality of the input current, making it smoother (see Figs. 16 and 17). The EMI noise generated is also reduced as a result (compared to a single module taking the same current).

Fig. 12 shows the simulation results for multiple disturbance harmonics on the DC bus. Here, until $t=0.372 \mathrm{~s}$ the test bench is generating a disturbance at $100 \mathrm{~Hz}$ with $200 \mathrm{~V}$ peak-to-peak ripple. At $t=0.372 \mathrm{~s}$ an additional $20 \mathrm{~A}$ disturbance current at $170 \mathrm{~Hz}$ is added on the DC bus. The MAC suppresses this disturbance with multiple harmonics and maintains a low ripple of less than $10 \mathrm{~V}$ peak-to-peak.

\section{B. Multiple MACs in a Microgrid}

In this section we show the simulation results for multiple MACs connected at different nodes in a DC microgrid. One typical application can be found in hybrid AC/DC microgrids [33]. The simulation setup is shown in Fig. 13. The ripple source converter is a single-phase AC/DC converter, regulating the DC bus at $390 \mathrm{~V}$ while generating large second harmonics. Three MACs are distributed, connected by cables of different length: $l_{01}$ $=10 \mathrm{~m}, l_{02}=100 \mathrm{~m}, l_{23}=l_{13}=200 \mathrm{~m}$. The cable inductance is 0.85

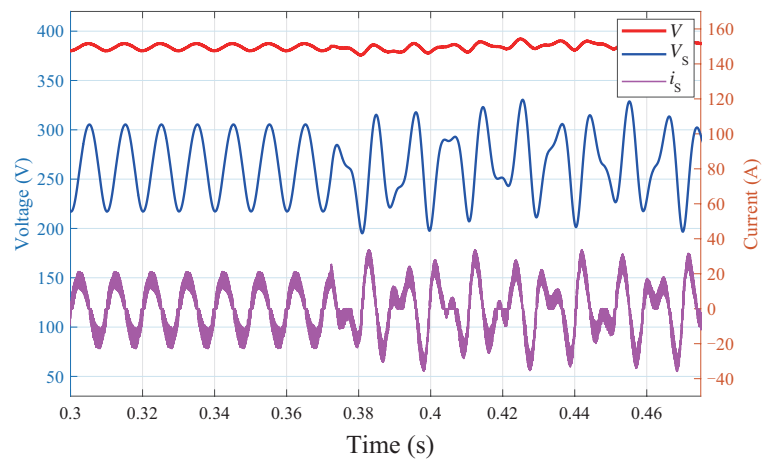

Fig. 12. Simulation results for multiple disturbance harmonics on the DC bus. A disturbance at $170 \mathrm{~Hz}$ is added to the existing disturbance of $100 \mathrm{~Hz}$ on the DC bus at $t=0.372 \mathrm{~s}$.

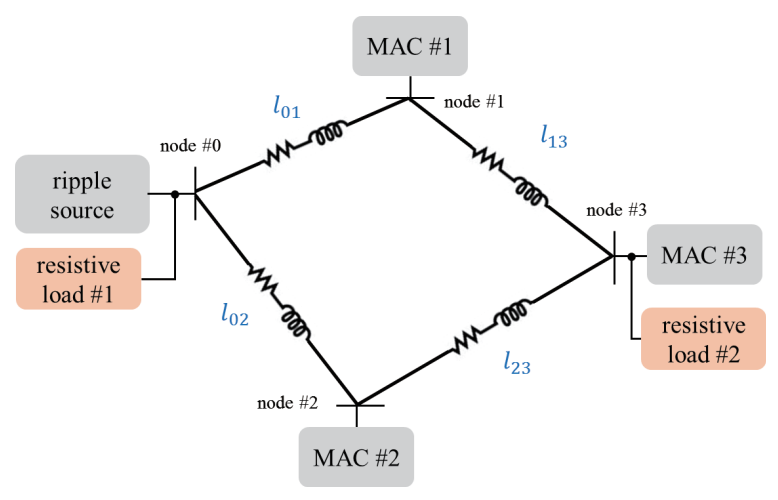

Fig. 13. The microgrid with three distributed MACs used in our simulations.

$\mathrm{mH} / \mathrm{km}$ and the cable resistance is $0.085 \Omega / \mathrm{km}$. Each MAC is regulated by its local controller, without communication between them. The load $R_{L 1}$ is $55 \Omega$ and $R_{L 2}$ is $250 \Omega$. To show the power sharing characteristics, the buffering capacitors for MAC \#2 and \#3 are set at $C_{\mathrm{S} 2,3}=100 \mu \mathrm{F}$ which is half that from MAC \#1, $C_{\mathrm{S} 1}=200 \mu \mathrm{F}$, and the inductor in MAC \#2 and \#3 is $L=56 \mu \mathrm{H}$ which is twice larger than in MAC \#1. Accordingly, the control gain $K_{0}$ for the power loop of MAC \#1 is twice larger.

Fig. 14 shows the transient when MAC \#2 is enabled (and MAC \#3 is not working). At the beginning, only $R_{L 1}$ is connected on the DC bus, and only MAC \#1 is working. At $t=0.7$ $\mathrm{s}$ (not shown in the figure), MAC \#2 is enabled. We can see the voltage waveforms of $V_{\mathrm{S} 1}$ and $V_{\mathrm{S} 2}$ slowly converge, and the current ratio $i_{\mathrm{S} 1} / i_{\mathrm{S} 2}=2$, in proportion to their storage capacity $C_{\mathrm{S} 1} / C_{\mathrm{S} 2}=2$.

In the first half of Fig. 15, all the three modules are working together to suppress the strong second harmonic. We disable MAC \#3 at $t=1.13 \mathrm{~s}$, representing a fault condition, then the ripples, which should be stored in MAC \#3, are shared in the other two modules in proportion, with almost no influence on the DC bus voltage.

\section{EXPERIMENTAL RESULTS}

\section{A. Performance of the MAC at Different Ripple Frequencies}

The experimental setup is shown in Fig. 9, with $N=3$. The 

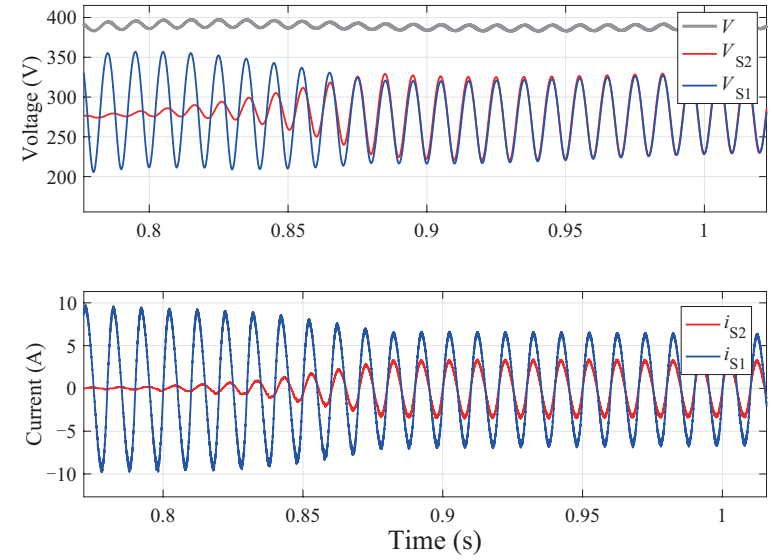

Fig. 14. Simulation results when MAC \#2 is enabled. MAC \#3 is not working. The lower subfigure shows the internal current $i_{\mathrm{S}}$ within MAC \#1 and MAC \#2, after a $1 \mathrm{KHz}$ low pass filter.
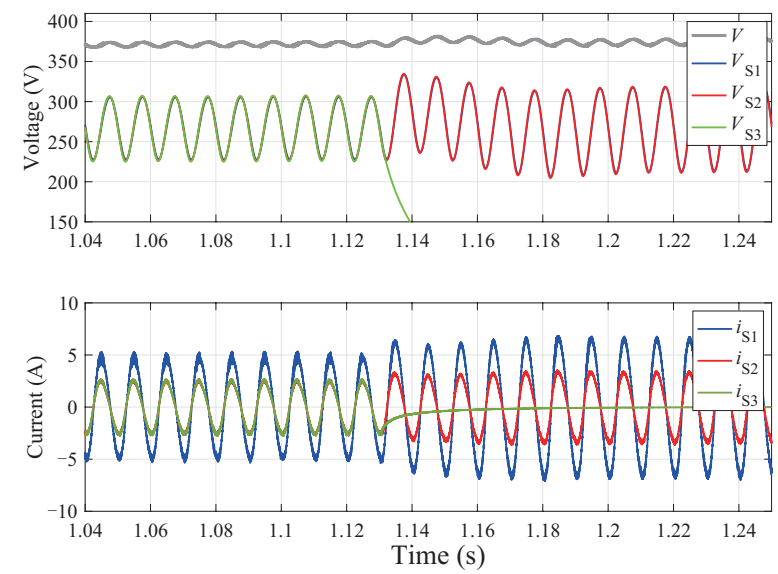

Fig. 15. Simulation results when MAC \#3 is disabled, representing a fault. The ripple currents are redistributed among MAC \#1 and \#2. Since $C_{\mathrm{S} 2}=C_{\mathrm{S} 3}$, their current $i_{\mathrm{S} 2}$ and $i_{\mathrm{S} 3}$ are identical in the first half of the figure.

response of the MAC to different $\mathrm{DC}$ bus disturbance frequencies ranging from $70 \mathrm{~Hz}$ to $300 \mathrm{~Hz}$ has been evaluated. An equivalent capacitance can be calculated using the formula

$$
C_{\text {eq }}=\frac{\Delta i}{\omega \Delta V},
$$

where $\Delta i$ is the ripple current, $\omega$ is the frequency and $\Delta V$ is the ripple on the DC bus voltage $V$. The results are listed in Table III, where the last column shows the capacitance reduction ratio (equivalent capacitance divided by $N\left(C+C_{\mathrm{S}}\right)$ ).

Here we show the experimental results for the DC bus disturbance frequencies of $70 \mathrm{~Hz}, 100 \mathrm{~Hz}$. In Figs. 16 and 17, the meaning of the plots is coded by color:

- channel 1 (blue): voltage across the buffering capacitor, $V_{\mathrm{S}}[100 \mathrm{~V} / \mathrm{div}]$,

- channel 2 (turquoise): DC bus voltage (with a DC offset), $V[10 \mathrm{~V} /$ div $]$,

- channel 3 (purple): total current $i_{\mathrm{S} \text {, tot }}$ flowing through the buffering capacitor $C_{\mathrm{S}, \text { tot, }}$
TABLE III

Performance at Different Freqencies

\begin{tabular}{lcccc}
\hline \hline $\begin{array}{l}\text { Ripple freq. } \\
f(\mathrm{~Hz})\end{array}$ & $\begin{array}{l}\Delta V \\
(\mathrm{~V})\end{array}$ & $\begin{array}{l}\Delta i \\
(\mathrm{~A})\end{array}$ & $\begin{array}{c}C_{\text {eq }} \\
(\mu \mathrm{F})\end{array}$ & $\begin{array}{l}\text { Normalized } \\
\text { by } C+C_{\mathrm{S}}\end{array}$ \\
\hline 70 & 3.4 & 4.4 & 2943.9 & 4.3 \\
80 & 3.4 & 5.2 & 3044.2 & 4.4 \\
90 & 2.8 & 6.6 & 4170.5 & 6.0 \\
100 & 2.8 & 9.0 & 5118.3 & 7.4 \\
150 & 2 & 9.0 & 4777.1 & 6.9 \\
200 & 1.8 & 9.0 & 3980.9 & 5.8 \\
300 & 1.6 & 8.8 & 2919.3 & 4.2 \\
\hline \hline
\end{tabular}

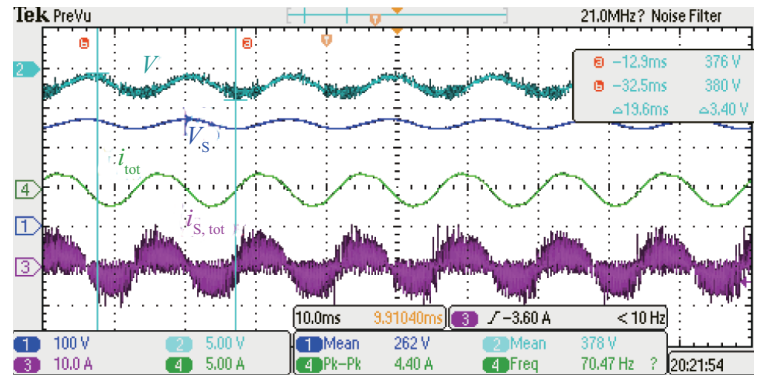

Fig. 16. Experimental results: the plots of $V, V_{\mathrm{S}}, i_{\mathrm{tot}}$ and $i_{\mathrm{S}, \mathrm{to}}$, arranged from top to bottom, the $\mathrm{DC}$ bus mean is $378 \mathrm{~V}$ with the disturbance ripple at $70 \mathrm{~Hz}$ and the ripple current $\Delta i_{\text {tot }}=4.4 \mathrm{~A}$. The peak-to-peak ripple on DC bus is $\Delta V=3.4 \mathrm{~V}$.

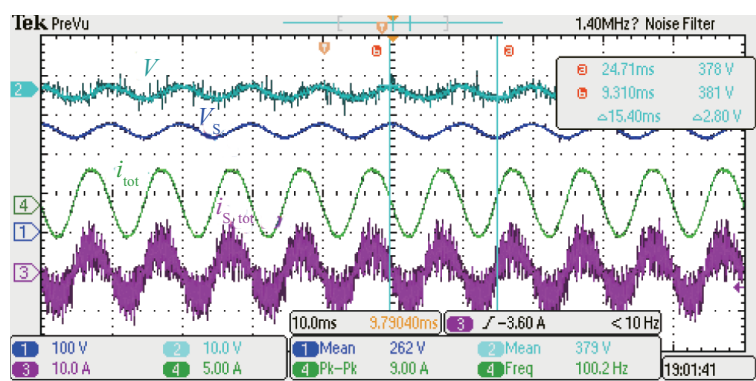

Fig. 17. Experimental results: the plots of $V, V_{\mathrm{S}}, i_{\mathrm{tot}}$ and $i_{\mathrm{S}, \mathrm{tot}}$, arranged from top to bottom, the DC bus mean is $379 \mathrm{~V}$ with the disturbance ripple at $100 \mathrm{~Hz}$ and the ripple current $\Delta i_{\mathrm{tot}}=9 \mathrm{~A}$. The peak-to-peak ripple on the DC bus is $\Delta V=2.8 \mathrm{~V}$.

- channel 4 (green): current flowing into the MAC, $i_{\text {tot }}$.

It can be seen clearly from the figures that the MAC is able to maintain a low ripple of less than $5 \mathrm{~V}$ peak-to-peak over a wide range of ripple frequencies, with the ripple currents $\Delta i$ in each case given in the third column of Table III. The interleaved inductor current waveforms of the individual phases in the MAC can be seen in Fig. 18. This is a snapshot taken at the moment when $i_{\text {tot }} \approx 1.2 \mathrm{~A}$.

\section{B. Load Change Experiments}

Figs. 19 and 20 show the experimental results for the load variation tests on the $\mathrm{DC}$ bus. The load $R_{L}$ is connected/disconnected manually using the switch $S W$ to simulate the load change, see Fig. 9. This causes a change (up or down) of about $15 \mathrm{~V}$ in the average value of the DC bus. This is because there is no feedback control for the test bench buck converter, the 


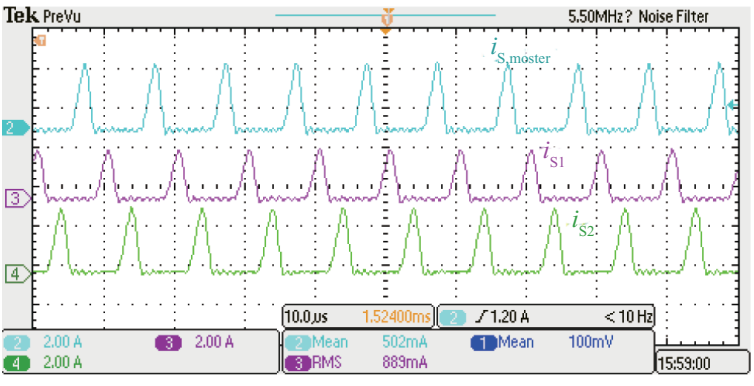

Fig. 18. Interleaved phase currents of the master and slave modules $i_{\mathrm{S} \text {, master, }} i_{\mathrm{S} 1}$ and $i_{\mathrm{S} 2}$, from top to bottom.

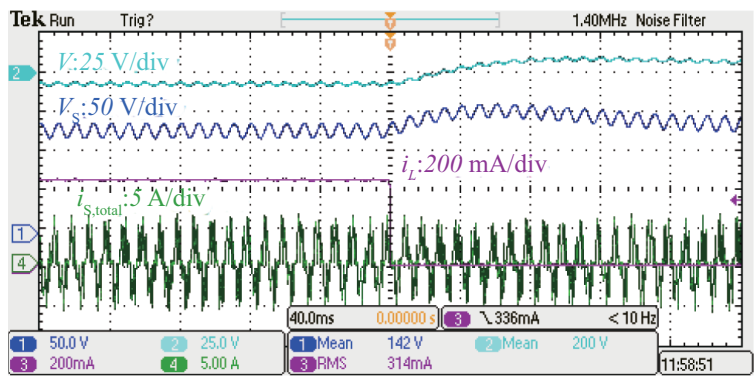

Fig. 19. Load variation experimental results for a MAC showing, from top (i) the DC bus voltage $V$; (ii) the buffering capacitor voltage $V_{\mathrm{S}}$; (iii) the load current $i_{L}$ and (iv) the total current $i_{\mathrm{S} \text {, tot }}$ when the load on the DC bus is changed from full load to no load.

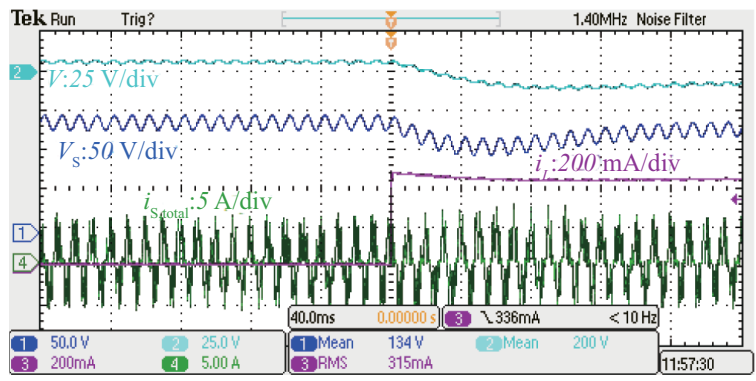

Fig. 20. Load variation experimental results for the MAC showing, from top (i) the DC bus voltage $V$; (ii) the buffering capacitor voltage $V_{\mathrm{S}}$; (iii) the load current $i_{L}$ and (iv) the total current $i_{\mathrm{S} \text {, tot }}$ when the load on the DC bus is changed from no load to full load.

duty cycle of the buck is controlled according to (10). However, it is important to notice that the DC bus voltage ripple remains within $5 \mathrm{~V}$ peak-to-peak before and after the sudden load decrease/increase.

\section{Multiple MACs in a DC Microgrid}

In Fig. 21, we show the experimental results for two MACs with equal buffering capacitors $C_{\mathrm{S}}$ connected to a common DC bus with a small inductor of $50 \mu \mathrm{H}$ between them. The 50 $\mu \mathrm{H}$ inductor emulates the line impedance of a microgrid. We can see that the buffering capacitor voltages of the two MACs, represented by the second and third waveforms from the top in Fig. 21, are synchronized.

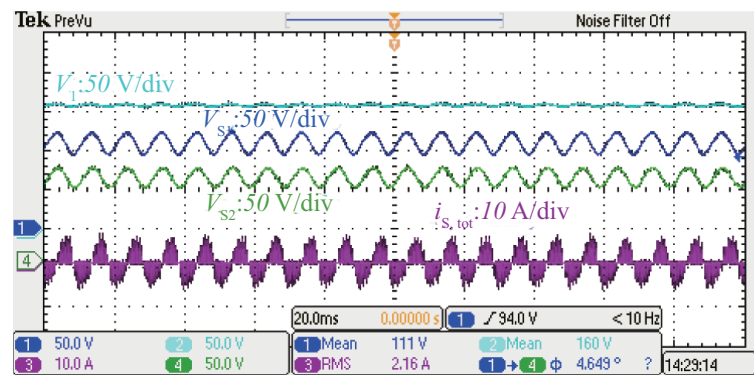

Fig. 21. Experimental results for two MACs separated by an inductor showing the waveforms from top (i) the DC bus voltage $V_{1}$ of MAC \#1; (ii) the buffering capacitor voltage for MAC \#1, $V_{\mathrm{S1}}$; (iii) the buffering capacitor voltage for MAC $\# 2, V_{\mathrm{S} 2}$; (iv) the total current $i_{\mathrm{S} \text {, tot }}$ for MAC \#1.

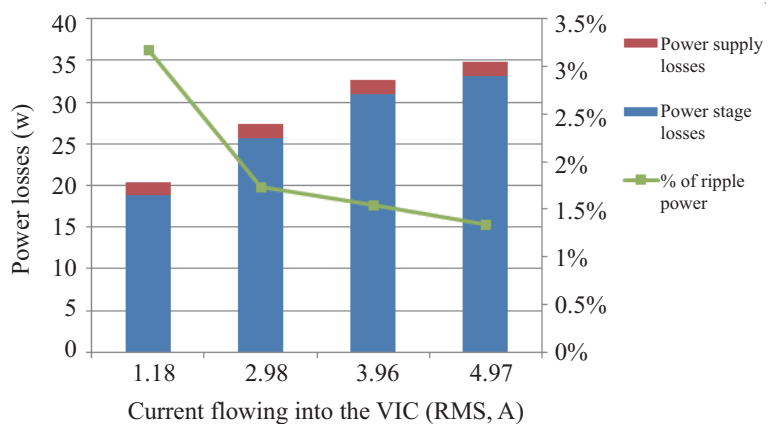

Fig. 22. Power losses chart.

\section{Power Losses}

To measure the power losses within the MAC, a $0.4 \Omega$ resistor is put between the grounds of the MAC and test bench. We measure the DC components of the voltage drop across this small resistor via a very low bandwidth low pass filter. The following data is measured under $380 \mathrm{~V}$ DC bus voltage, when $100 \mathrm{~Hz}$ ripple is injected. From Fig. 22, we can notice that the power losses is only a small portion of total ripple power.

\section{CONCLUSIONS}

In this paper we have proposed a modular realization of an active capacitor meant for DC bus voltage filtering in high power applications. The proposed two terminal MAC can be connected directly to the DC bus like a passive capacitor and is capable of adapting to the changing DC bus voltage and filter the disturbances in a wide range of frequencies. The interleaving technique used helps in reducing the EMI and the stresses on the active and passive components of the MAC. This paper also gives ideas on how to tune the MAC controller for higher frequencies and on how to choose the buffering capacitor $C_{\mathrm{S}}$ and the inductor $L$, depending on the expected ripple currents. We have tested the MAC in simulations and experiments, also covering the case of multiple MACs in a DC microgrid.

\section{REFERENCES}

[1] X. Huang, X. Ruan, F. Du, F. Liu, and L. Zhang, "A pulsed power supply 
adopting active capacitor converter for low-voltage and low-frequency pulsed loads," in IEEE Transactions on Power Electronics, vol. 33, no. 11, pp. 9219-9230, Nov. 2018.

[2] X. Cao, Q. -C. Zhong, and W. -L. Ming, "Ripple eliminator to smooth dcbus voltage and reduce the total capacitance required," in IEEE Transactions on Industrial Electronics, vol. 62, no. 4, pp. 2224-2235, Apr. 2015.

[3] G. Yona and G. Weiss, "The virtual infinite capacitor," in International Journal of Control, vol. 90, pp. 78-89, 2017.

[4] J. Lin and G. Weiss, "An indirect approach to control an active capacitor," in IEEE Journal of Emerging and Selected Topics in Power Electronics, vol. 8, no. 3, pp. 2898-2906, Sept. 2020.

[5] J. Lin, "Decentralized control of an active capacitor with NDO-based feedforward compensation," in IEEE Journal of Emerging and Selected Topics in Power Electronics, vol. 8, no. 4, pp. 3960-3967, Dec. 2020.

[6] Y. Sun, Y. Liu, M. Su, W. Xiong, and J. Yang, "Review of active power decoupling topologies in single-phase systems," in IEEE Transactions on Power Electronics, vol. 31, no. 7, pp. 4778-4794, Jul. 2016.

[7] M. A. Vitorino, L. F. S. Alves, R. Wang, and M. B. de Rossiter Correa, "Low-frequency power decoupling in single-phase applications: A comprehensive overview," in IEEE Transactions on Power Electronics, vol. 32, no. 4, pp. 2892-2912, Apr. 2017.

[8] H. Wang, H. Wang, G. Zhu, and F. Blaabjerg, "An overview of capacitive DC-links-topology derivation and scalability analysis," in IEEE Transactions on Power Electronics, vol. 35, no. 2, pp. 1805-1829, Feb. 2020.

[9] Z. Qin, Y. Tang, P. C. Loh, and F. Blaabjerg, "Benchmark of AC and DC active power decoupling circuits for second-order harmonic mitigation in kilowatt-scale single-phase inverters," in IEEE Journal of Emerging and Selected Topics in Power Electronics, vol. 4, no. 1, pp. 15-25, Mar. 2016.

[10] D. Neumayr, D. Bortis, and J. Kolar, "Ultra-compact power pulsation buffer for single-phase DC/AC converter systems," in Proceedings of 2016 IEEE 8th International Power Electronics and Motion Control Conference, Hefei, China, May 2016, pp. 2732-2741.

[11] J. M. Alonso, J. Vina, D. G. Vaquero, G. Martinez, and R. Osorio, "Analysis and design of the integrated double buck-boost converter as a high-power-factor driver for power-LED lamps," in IEEE Transactions on Industrial Electronics, vol. 59, no. 4, pp. 1689-1697, Apr. 2012.

[12] H. Watanabe, T. Sakuraba, K. Furukawa, K. Kusaka and J. Itoh, "Development of DC to single-phase AC Voltage source inverter with active power decoupling based on flying capacitor DC/DC converter," in IEEE Transactions on Power Electronics, vol. 33, no. 6, pp. 4992-5004, Jun. 2018.

[13] S. Li, W. Qi, S. Tan, and S. -Y. -R. Hui, "A single-stage two-switch PFC rectifier with wide output voltage range and automatic AC ripple power decoupling," in IEEE Transactions on Power Electronics, vol. 32, no. 9, pp. 6971-6982, Sept. 2017.

[14] R. Wang, F. Wang, D. Boroyevich, R. Burgos, R. Lai, P. Ning, and K. Rajashekara, "A high power density single-phase PWM rectifier with active ripple energy storage," in IEEE Transactions on Power Electronics, vol. 26, no. 5, pp. 1430-1443, May 2011.

[15] H. Yuan, S. Li, W. Qi, S. C. Tan, and R. Hui, "On nonlinear control of single-phase converters with active power decoupling function," in IEEE Transactions on Power Electronics, vol. 34, no. 6, pp. 5903-5915, 2018.

[16] K. Sharifabadi, L. Harnefors, H. -P. Nee, S. Norrga, and R. Teodorescu, in Design, Control, and Application of Modular Multilevel Converters for HVDC Transmission Systems, Wiley-IEEE Press, Oct. 2016.

[17] S. Li, W. Qi, S. Tan, S. Y. Hui, and C. K. Tse, "A general approach to programmable and reconfigurable emulation of power impedances," in IEEE Transactions on Power Electronic, vol. 33, no. 1, pp. 259-271, Jan. 2018.

[18] M. Mellincovsky, V. Yuhimenko, M. Peretz, and A. Kuperman, "Low frequency DC-link ripple elimination in power converters with reduced capacitance by multiresonant direct voltage regulation," in IEEE Transactions on Industrial Electronics, vol. 64, no. 3, pp. 2015-2023, Mar. 2017.

[19] S. Li, A. Lee, S. Tan, and S. Hui, "Plug-and-play voltage ripple mitigator for DC links in hybrid AC-DC power grids with local bus-voltage control," in IEEE Transactions on Industrial Electronics, vol. 65, no. 1, pp. 687-698, Jan. 2018.

[20] H. Wang and H. Wang, "A two-terminal active capacitor," in IEEE Transactions on Power Electronics, vol. 32, no. 8, pp. 5893-5896, Aug. 2017.
[21] J. Lin and G. Weiss, "Plug-and-play control of the virtual infinite capacitor," in IEEE Transactions on Power Electronics, vol. 35, no. 2, pp. 1947-1956, Feb. 2020

[22] A. Mutovkin, V. Yuhimenko, M. Mellincovsky, S. Schacham and A. Kuperman, "Control of direct voltage regulated active DC-link capacitance reduction circuits to allow plug-and-play operation," in IEEE Transactions on Industrial Electronics, vol. 66, no. 8, pp. 6527-6537, Aug. 2019.

[23] J. Lin and G. Weiss, "The virtual infinite capacitor-based active submodule for MMC," in Proceedings of 2016 IEEE International Conference on the Science of Electrical Engineering (ICSEE), Eilat, Israel, 2016, pp. 1-5.

[24] O. Hegazy, J. V. Mierlo, and P. Lataire, "Analysis, modeling, and implementation of a multidevice interleaved DC/DC converter for fuel cell hybrid electric vehicles," in IEEE Transactions on Power Electronics, vol. 27, no. 11, pp. 4445-4458, Nov. 2012.

[25] L. Ni, D. J. Patterson, and J. L. Hudgins, "High power current sensorless bidirectional 16-phase interleaved DC/DC converter for hybrid vehicle application," in IEEE Transactions on Power Electronics, vol. 27, no. 3, pp. 1141-1151, Mar. 2012.

[26] J. Zhang, J. Lai, R. Kim, and W. Yu, "High-power density design of a soft-switching high-power bidirectional DC/DC converter," in IEEE Transactions on Power Electronics, vol. 22, no. 4, pp. 1145-1153, Jul. 2007.

[27] Y. Lee, Y. Ko, M. Cheng, and L. Liu, "Multiphase zero-current switching bidirectional converters and battery energy storage application," in IEEE Transactions on Power Electronics, vol. 28, no. 8, pp. 3806-3815, Aug. 2013.

[28] D. R. Garth, W. J. Muldoon, G. C. Benson, and E. N. Costague, "Multiphase, 2 kilowatt, high voltage, regulated power supply", in Proceedings of 1971 IEEE Power Electronics Specialists Conference, Pasadena, CA, USA, 1971, pp. 110-116.

[29] S. Ozeri, D. Shmilovitz, S. Singer, and L. Martinez-Salamero, "The mathematical foundation of distributed interleaved systems", in IEEE Transactions on Circuits and Systems I: Regular Papers, vol. 54, no. 3, pp. 610-619, Mar. 2007.

[30] Z. Yuan and H. Xu, "Pulse power supply with faster response and low ripple current using inductive storage and interleaving technology," in CPSS Transactions on Power Electronics and Applications, vol. 5, no. 1, pp. 54-62, Mar. 2020.

[31] C. Guiver, H. Logemann, and M. Opmeer, "Transfer functions of infinitedimensional systems: Positive realness and stabilization," in Mathematics of Control Signals and Systems, vol. 29, no. 4, Dec. 2017.

[32] R. B. Burckel, in An Introduction to Classical Complex Analysis, vol. 1, New York: Academic Press, Inc., 1979.

[33] H. Tian and Y. Li, "Virtual resistor based second-order ripple sharing control for distributed bidirectional DC-DC converters in hybrid ACDC microgrid," in IEEE Transactions on Power Electronics, vol. 36, no. 2, pp. 2258-2269, 2021.

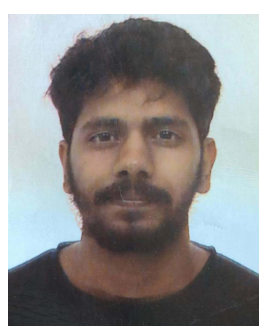

Vinay Bhus received the B.Sc. degree in electrical and electronics engineering from Tel Aviv University, $\mathrm{Tel}$ Aviv, Israel, in 2018, and the M.Sc. degree in power electronics at Tel Aviv University in 2021. He is currently working at an electro-optics company in Israel. 


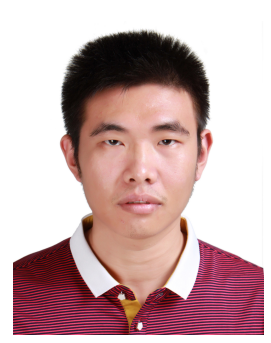

Jyun Lin received the B.Sc. degree in mechanical engineering from the University of Yantai, China, in 2008, and the dual M.Sc. degree in automation and control engineering-embedded systems from Politecnico di Milano, Milan, Italy, and ISAE-SUPAERO, University of Toulouse, Toulouse, France, in 2013 and 2014, respectively. He has recently completed the Ph.D. in Electrical Engineering at Tel Aviv University, Israel, and has taken up a post-doctoral position at the Technical University of Denmark, in the research group of Prof. Tomislav Dragicevic.

His research interests include control techniques applied to power electronics, and mechatronics.

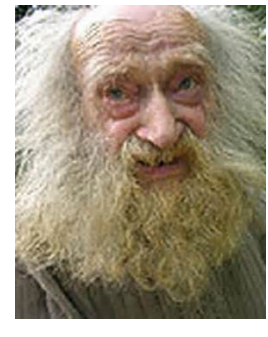

George Weiss received the M.Eng. degree in control engineering from the Polytechnic Institute of Bucharest, Bucharest, Romania, in 1981, and the Ph.D. degree in applied mathematics from Weizmann Institute, Rehovot, Israel, in 1989.

He was with Brown University, Providence, RI, USA, Virginia Polytechnic Institute and State University, Blacksburg, VA, USA, Ben-Gurion University, Beer Sheva, Israel, University of Exeter, Exeter, U.K., and Imperial College London, London, U.K. He is currently a Professor of control engineering with Tel Aviv University, Tel Aviv, Israel. His current research interests include distributed parameter systems, operator semigroups, passive and conservative systems (linear and nonlinear), power electronics, repetitive control, sampled data systems, and wind-driven power generators.

\section{Statement of Correct Errors}

The following published paper of CPSS TPEA need to be corrected here.

1. Title: Comprehensive Analysis and Design of LLC Resonant Converter With Magnetic Control

2. Authors: Yuqi WEI, Quanming LUO, and Alan MANTOOTH

3. Published Information: vol. 4, no. 4, pp. 265-275, December 31, 2019

4. DOI: $10.24295 /$ CPSSTPEA.2019.00025

5. Details: Due to the oversight of authors, Fig. 2(a) in the paper had missed a citation to the original work. The caption should have referenced this paper as follows:

[30] D. Medini and S. Ben-Yaakov, "A current-controlled variable-inductor for high frequency resonant power circuits," in Proceedings of 1994 IEEE Applied Power Electronics Conference and Exposition - APEC'94, Orlando, FL, USA, vol. 1, Feb. 1994, pp. 219-225.

Editorial Office

CPSS Transactions on Power Electronics and Applications

September 30, 2021 